\title{
Lipiodol lymphangiography as a treatment for refractory postoperative chylothorax: a case report
}

\author{
Nesrine Lamine, Apostolos C. Agrafiotis, Ines Lardinois \\ Thoracic Surgery Division, Saint-Pierre University Hospital, Université Libre de Bruxelles (ULB), Brussels, Belgium
}

\begin{abstract}
Postoperative chylothorax is the most frequent cause of traumatic thoracic chyle leakage. Conservative treatment is primarily used to treat low flow rate chylothoraces and should be initiated as soon as the diagnosis has been made. In case of high flow rate chylothorax or failure of initial treatment, surgery and radiological treatment are indicated. Despite this fact, there is a lack of consensus regarding the best therapeutic option to choose. In the case reported herein, a postoperative chylothorax with a low flow chyle leakage which didn't respond to conservative treatment is demonstrated. We used lipiodol lymphangiography to determine the site of chyle leakage and to successfully treat our patient. Risk factors for non-response to conservative treatment are also discussed.
\end{abstract}

Correspondence: Nesrine Lamine, Rue Haute 322, 1000 Brussels, Belgium.

Tel. +32.2535.3111. E-mail: nesrine.lamine@ulb.be

Authors' contributions: NL, literature research and article drafting; ACA, literature research, manuscript appraisal; IL, final approval.

Conflict of interest: The authors declare no potential conflict of interest.

Informed consent: Informed consent was obtained from the patient for data and image use.

Funding: None.

Availability of data and materials: All data generated or analyzed during this study are included in this published article.

Key words: Post-operative chylothorax; medium-chain triglycerides diet; thoracic duct; lipiodol lymphangiography.

Received for publication: 11 November 2020.

Accepted for publication: 28 April 2021.

${ }^{\circ}$ Copyright: the Author(s), 2021

Licensee PAGEPress, Italy

Monaldi Archives for Chest Disease 2021; 91:1676

doi: 10.4081/monaldi.2021.1676

This article is distributed under the terms of the Creative Commons Attribution Noncommercial License (by-nc 4.0) which permits any noncommercial use, distribution, and reproduction in any medium, provided the original author(s) and source are credited.

\section{Introduction}

Chylothorax is a chylous pleural effusion that has multiple etiologies and is usually classified as traumatic and nontraumatic [1]. The most frequent cause of traumatic chyle leakage is post-operative and concerns different types of surgery as esophageal, cardiac, aortic, pulmonary, neck, sympathetic nerve, and diaphragmatic operations [2].

In the particular case of lung resections, right-sided lobectomies, robotic approach and pathologic N2 disease are considered risk factors for the development of postoperative chylothoraces [3]. Initial treatment is conservative (pleural drainage, lowfat diet, somatostatin or its derivatives and total parenteral nutrition). More invasive procedures, such as surgery and interventional radiology are indicated for chylothoraces with high daily output $(>1 \mathrm{~L})$ and in cases where conservative approach fails after a reasonable amount of time [4]. However, there is no consensus when and for how long a treatment should be considered effective.

In the case reported herein, we will present a chylothorax following lung cancer surgery, which was refractory to conservative treatment, and try to identify potential risk factors for conservative treatment failure.

\section{Case report}

A 68-years-old male patient underwent a right upper sleeve lobectomy combined with a S6 segmentectomy and radical mediastinal lymph node dissection for a primary lung adenocarcinoma after four cycles of neoadjuvant chemotherapy (carboplatin-pemetrexed).

The patient's medical history included the surgical treatment of a squamous cell carcinoma of the base of the tongue associated with bilateral radical neck dissection with reconstruction.

After lung resection, a milky fluid appeared in the chest tube on the second postoperative day. The diagnosis of chylothorax was confirmed by biochemistry analysis. Pleural fluid triglyceride was $1310 \mathrm{mg} / \mathrm{dL}$ and cholesterol $79 \mathrm{mg} / \mathrm{dL}$ (cut-off values for triglyceride concentration $>110 \mathrm{mg} / \mathrm{dL}$ diagnostic for chylothorax and cholesterol concentration more than $200 \mathrm{mg} / \mathrm{dL}$ suggestive of pseudochylothorax or cholesterol effusion) [2].

A medium-chain triglycerides (MCT) diet was initiated. However, two weeks after treatment, chyle leakage has not been reduced and it has been decided to treat the patient with complete parenteral nutrition in adjunction to somatostatin analogues (subcutaneous injections). Because there was still no improvement a week later and because the patient was not willing to undergo surgery for thoracic duct ligation, lipiodol lymphangiography was 


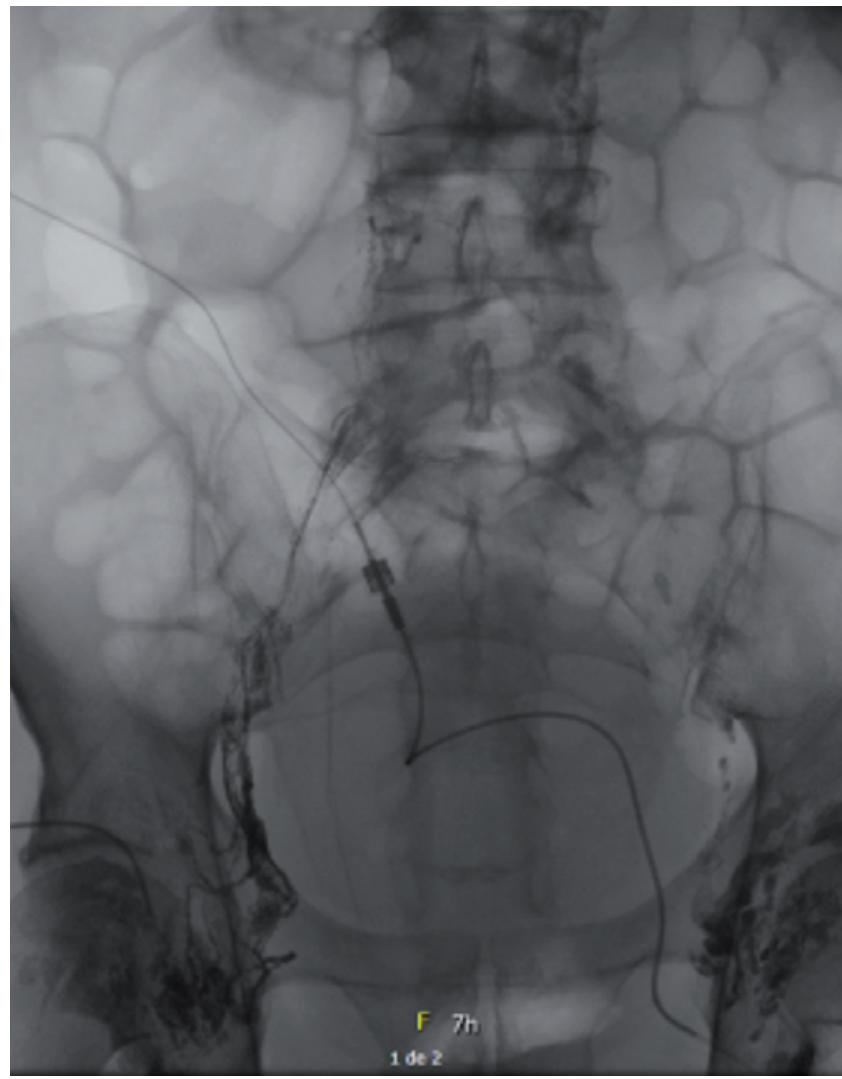

Figure 1. Lipiodol lymphangiography. Inguinal lymph node puncture.

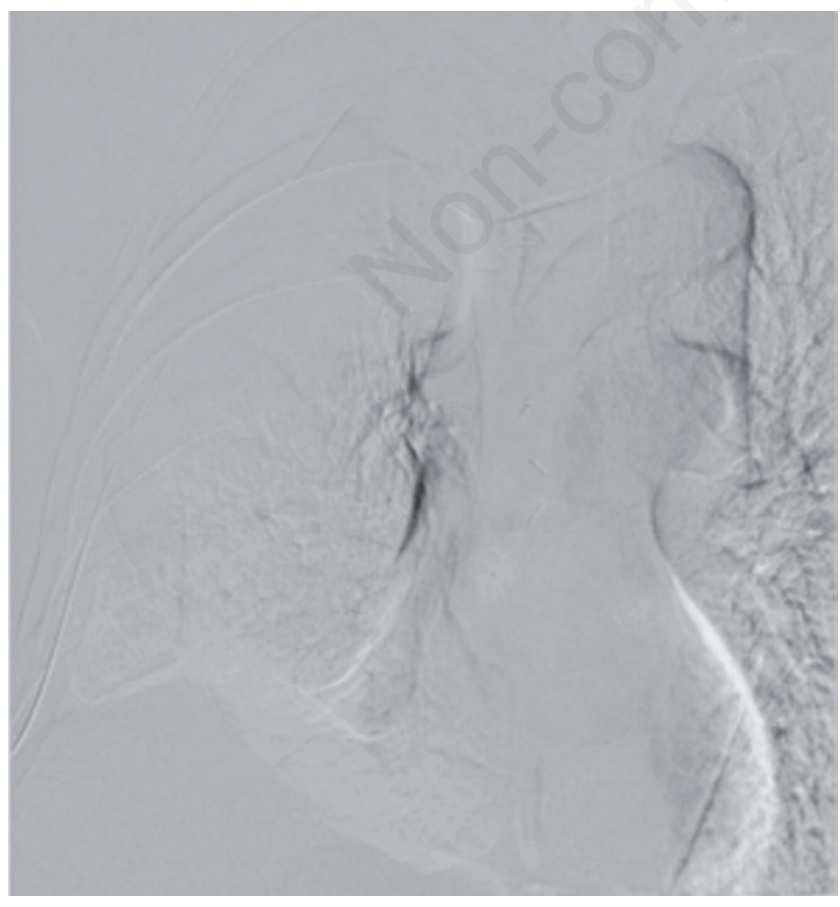

Figure 2. Lipiodol lymphangiography. Lipiodol right paratracheal extravasation. performed. An inguinal lymph node was punctured with echography guidance and lipiodol was injected (Figure 1). A contrast extravasation suggesting a right paratracheal lymph leak has been identified (Figure 2). In order to perform an embolization of the thoracic duct, a percutaneous puncture of the right cisterna chyli has been planned, nevertheless its catheterization was technically not possible.

One week later, a second lymphangiography was performed in order to inject more of the sclerosing agent; this time there was no contrast extravasation (Figure 3).

Two days following the second exam, the daily quantity of fluid in the chest tube was considerably decreased. Complete parenteral nutrition has been stopped. The pleural drain was removed without recurrence of the effusion and the patient was discharged.

\section{Discussion}

Various options are available in order to treat postoperative chylothorax, nutritional interventions and chest drainage to decrease chyle production are usually used initially. Conservative treatment is variably effective from $50 \%$ to $80 \%$ success rate in postoperative chylothorax [5].

In the case reported herein, since the daily leakage volume was low (under $600 \mathrm{~mL}$ per day) the conservative treatment was expected to be sufficient. However, it was not efficacious after several days resulting in long term hospital stay, diet restriction, use of parenteral nutrition and chest drainage. The reason why in this case the chylothorax was refractory to the conservative treatment has not been elucidated [6,7]. Risk factors for failed attempts to standard approach has not yet been clearly identified.

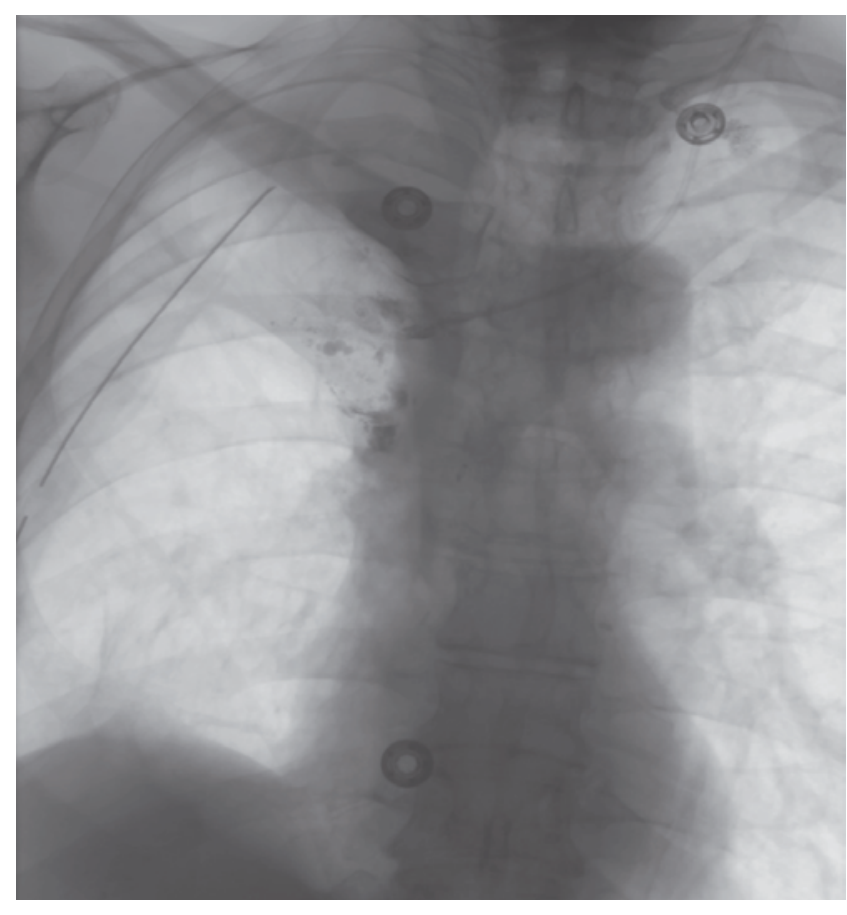

Figure 3. Lipiodol lymphangiography. Thoracic lymphatic opacification without contrast extravasation. 
In this particular case we have speculated that the history of radical node dissection in the neck resulted in raised pressure into the lymphatic network and thus preventing healing of the incontinent lymphatic vessel that was injured during right paratracheal lymph node dissection.

Because the patient refused surgery for thoracic duct ligation, we used lymphangiography which is a well-known diagnostic tool used to identify the chylous leak, provide information about the anatomy of the lymphatic network and in certain cases to treat the chylothorax [8].

In our case, the chyle leakage ceased after the second tentative. This technique is easy to perform and has a high rate of clinical success $(60 \%$ to $80 \%)$ described as reduction in lymphatic drainage volume or complete cessation of chyle leak. In addition, it can identify the site of the leakage and thus provide important information for further surgical treatment [9]. The typical course of the thoracic duct which drains lymph running from the cisterna chyli to the left jugular vein has only been described in $40-60 \%$ of patients [10].

\section{Conclusions}

The success of conservative management of low volume postoperative chylothorax is highly variable. The consequences of untreated chylothorax are important: prolonged hospital stay, risk of infection, malnutrition, immunosuppression and quality of life impairment. Therefore, it will be interesting to identify the risk factors predicting failure of conservative treatment and discuss with the patient before surgery. Lymphangiography has a high rate of success, is less invasive compared with thoracic duct ligation, a cost effective and safe procedure. It should be part of the therapeutic armamentarium early in the post-operative course in case of failure of conservative treatment. Preoperative strategy should be tailored according to the presence of predisposing factors.

\section{References}

1. Cholet C, Delalandre C, Monnier-Cholley L et al. Nontraumatic chylothorax: non-enhanced MR lymphography. Radiographics 2020;40:1554-73.

2. Nair SK, Petko M, Hayward MP. Aetiology and management of chylothorax in adults. Eur J Cardiothorac Surg 2007;32: 362-9.

3. Bryant AS, Minnich DJ, Wei B, Cerfolio RJ. The incidence and management of post-operative chylothorax after pulmonary resection and thoracic mediastinal lymph node dissection. Ann Thorac Surg. 2014;98:232-7.

4. Schild HH, Strassburg CP, Welz A, Kalff J. Treatment options in patients with chylothorax. Dtsch Arztebl Int 2013;110:819.

5. Bender B, Murthy V, Chamberlain RS. The changing management of chylothorax in the modern era. Eur J Cardiothorac Surg 2016;49:18-24.

6. Kranzfelder M, Gertler R, Hapfelmeier A, et al. Chylothorax after esophagectomy for cancer: impact of the surgical approach and neoadjuvant treatment: systematic review and institutional analysis. Surg Endosc 2013;27:3530-8.

7. Ohkura Y, Ueno M, Shindoh J et al. Risk factors for postoperative chylothorax after radical subtotal esophagectomy. Ann Surg Oncol 2018;25:2739-2746.

8. Suetsugu T, Tanaka Y, Banno S, et al. Intranodal lymphangiography for chyle leakage after esophagectomy: A case report. Mol Clin Oncol 2020;12:343-349.

9. Lee KH, Jung JS, Cho SB, et al. Thoracic duct embolization with lipiodol for chylothorax due to thoracic endovascular aortic repair with debranching procedure. Korean J Thorac Cardiovasc Surg 2015;48:74-7.

10. Skandalakis JE, Skandalakis LJ, Skandalakis PN. Anatomy of the lymphatics. Surg Oncol Clin N Am 2007;16:1-16. 